\title{
A Robotized Positioning Platform Guided by Computed Tomography : Practical Issues and Evaluation
}

\author{
B. Maurin, B. Bayle, J. Gangloff, P. Zanne, M. de Mathelin \\ LSIIT, UMR ULP-CNRS 7005 \\ Pole API, Bd. S. Brant, 67412 Illkirch, France \\ maurin@eavr.u-strasbg.fr
}

\author{
Olivier Piccin \\ LGeCo, INSA-Strasbourg \\ 67084 Strasbourg, France
}

\begin{abstract}
Medical robotics is a field where dedicated mechanisms have an increasing importance. The strong operating room constraints, both medical and practical, lead to heavily customized solutions. In this paper, we consider the practical problems that must be solved to build a robotic system dedicated to medical interventions under CT-scan guidance. This compact robotic assistant is placed on the patient what raises new generic robotic design problems. Practical solutions together with the evaluation of a prototype are presented in this paper.
\end{abstract}

\section{INTRODUCTION}

Whereas industrial manipulators have undoubtly reached a high degree of maturity, medical robotics applications recently opened a new field of investigation in manipulation. This corresponds to the development of dedicated mechanisms, adapted to both medical and practical constraints of the operating room. The robotic systems that have appeared can be considered as manipulators because of the tasks they are dedicated to. Nevertheless, these systems are completely different from standard manipulators. They are characterized by their compactness, lightness, good force/weight ratio, safety, sterility, and, of course, by their ability to accomplish a particular medical task with a noticeable improvement. Practical problems resulting from these constraints can be considered as quite generic. In this paper we will raise these robotic issues as generally as possible through the presentation of the solutions we used to build a robotic system dedicated to percutaneous interventions.

\section{A. Motivation}

Major breakthroughs in medical robotics have recently been achieved by visual guided robotic systems that use common medical imaging devices to localize themselves and execute a given task. While Computed Tomography (CT) is first of all a convenient and accurate indirect imaging technique for medical diagnosis, it is also appropriate for medical interventions. This is the case for percutaneous procedures which are commonly and successfully used for the treatment of chest or abdomen diseases [1]. They are less painful than open surgery and have many benefits: shorter time, accuracy, access to almost any organ, less trauma for the patient. These procedures consists in inserting a needle in the body of a patient, to reach a given target organ. Thanks to the CT-scan guidance, the radiologist can check the needle tip position to obtain a good accuracy, typically around $5 \mathrm{~mm}$ for an expert. The major drawback of these procedures is the $\mathrm{X}$-rays exposition of the radiologist during each procedure and the difficulty to incorporate navigation features in the operating room. X-rays exposition is even more harmful during CT fluoroscopy-guided interventions when very high accuracy is required [2].

The concept of holding a needle inside a CT-scan or a MRI device is not new. To our knowledge, [3] has initiated the first work on stereotactic CT-guided surgery. Since then, the RCM+PAKY system [4], [5], [6], [7] has proved its efficiency in the operating room. Other experiments in other fields, e.g. neurosurgery [8] and with other imaging modalities [9], showed that computer-aided needle insertions can be used in clinical cases.

A major difficulty in percutaneous treatments is due to the fact that the abdominal region moves with respect to the operation table when the patient breathes or accidentally coughs. To this day, the compensation of such biological movements in order to provide safety and high accuracy during needle insertions remains a challenging problem. To solve the problem of motion compensation, the concept of a robotic system placed on the patient body has been proposed in [10], [11], [9] and [12]. The system then moves with the patient, what prevents it from accidental motions. In that case, breathing is safely compensated without any special device.

In the following, we will deal with the practical issues resulting from positioning such a robot on the patient's body. To that purpose, we will consider a system similar to the one proposed in [12] that we briefly present in the following section.

\section{B. A compact robotic system placed on the patient}

The robotic system proposed in [12], called CT-Bot, is composed of a positioner and a needle driver. The robotic positioner is a small rigid parallel structure fastened to the abdomen of the patient with special straps. A pointing tool, a passive needle guide or a needle driver can be attached to the upper part of the device. Nevertheless, in the purpose of 
illustrating generic practical problems related to such a robot, we will focus on the positioner itself. The different devices that may be embedded on the platform are out of the scope of this paper.

The robotic system is a parallel structure made of a 6bar linkage associated to a 4-bar linkage joined together by a common platform (cf. Fig. 1). The mechanism has five degrees of freedom (DOF), three DOF for the position and two DOF for the orientation. Three DOF are imposed by the three actuators of the 6-bar linkage. The second linkage constrains the remaining DOF of the first linkage thanks to two other actuators. It results a 5 DOF parallel manipulator driven by five actuators.

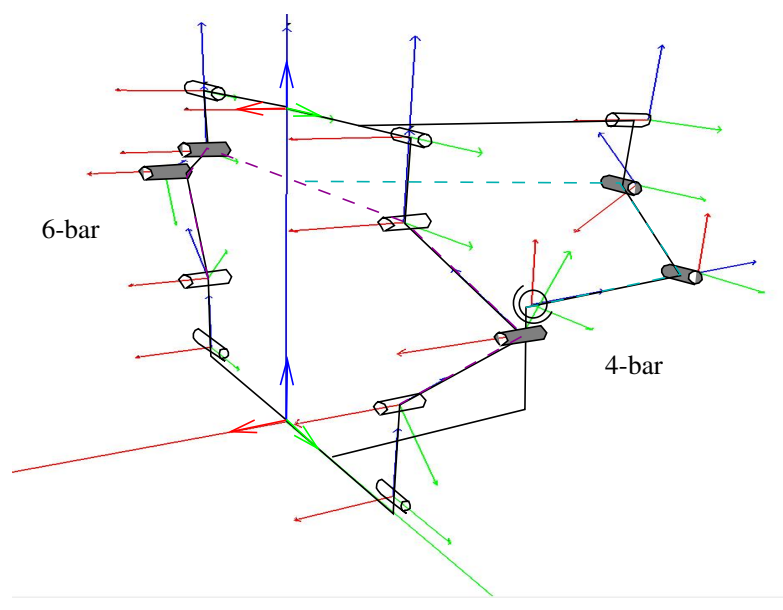

Fig. 1. Kinematic structure of the system

This system has the noticeable property of having analytical kinematic models [13]. Multiple solutions can be found either for the inverse kinematics ( 8 solutions) and for the forward kinematics (4 solutions). The particular choices made for the linkages lead to a simple geometric interpretation (see Fig. 1). The coupler of the 6-bar linkage spans a circle on which the orientation needs to be defined, and the 4-bar linkage defines the position of the coupler (distance to the spherical joint) and its orientation. The Jacobian matrix can been numerically computed using the first order derivatives of the kinematics.

\section{Contribution}

The justification and the modeling of the robotic system were previously published by the designers of the CT-Bot, principally in [13] and [12]. For that reason we won't make any further developments in that field. The registration of a system placed in the CT-scan ring from a single CT-slice has also been developed by different contributors [14], [15], [16]. This point will not be developed either, except in the experiments where some results depend on the registration quality.
The present paper will focus on the different specific points that have to be solved to make such a robotic concept work. In section II we will deal with the most noticeable practical issues, which might be reusable in future medical robotic systems. After this overview of the system technology, we will present in section III the methods we used to evaluate its performances and its ability to solve the tasks it has been designed for.

\section{A ROBOt ON THE PATIENT : PRACTICAL ISSUES}

\section{A. Design and manufacturing}

Placing a robot on a patient requires an optimal choice of size and weight. The space in the CT-scan ring is limited, specially for obese patients. A size less than $200 \mathrm{~mm}$ high, $250 \mathrm{~mm}$ large and $200 \mathrm{~mm}$ depth corresponds to the maximum limit for a large range of patients. Since the system is placed on the patient, its weight has also to be limited. The prototype we realized is less than $2.5 \mathrm{Kg}$. Thus, the robotic system is characterized by its compactness. To that purpose, most parts of the robot were obtained with polymer rapid prototyping. The parts that ensure the system rigidity, particularly the robot links, are the only metallic ones. They have all been placed in order to avoid intersections with the CT-scan plane which could yield artefacts in the image.

To adapt to different tasks and patients, some improvements have been made at the interface between the body and the robot base. First, a robot base support is attached with straps on the patient body. Since this contact corresponds to the interface between soft tissues and the rigid base, it is likely to generate a lack of stability for the robot. To prevent that, we designed an interface made of a special vacuum mattress. This mattress is filled with tiny balls of polymer and can be shaped accordingly to the surface of the patient's body. When the air is pumped out, the mattress becomes rigid and so ensures an optimal contact between the skin and the base of the robot.

The placement of the robot is a 3-stages procedure. First, the base is placed purposely on the patient thanks to radioopaque markers that are visible in the CT-scan image [16]. Then, when the base is positioned, the vacuum mattress is emptied in order to realize a comfortable and quite rigid connection between the robot base and the patient. Finally, the robot itself is oriented and fixed on the base support. This allows to choose the best initial configuration according to the intervention objective. A CAD drawing illustrating the whole system design is given on Fig. 2 .

\section{B. Driving system, position servoing and calibration}

The design of the motor and transmission of the system is a trade-off between safety, force and velocity considerations.

1) Motors: Since the system is placed on the patient's body the most important feature is of course safety. The minimum requirement linked to safety is that the system may stay naturally still in case of power failure. 


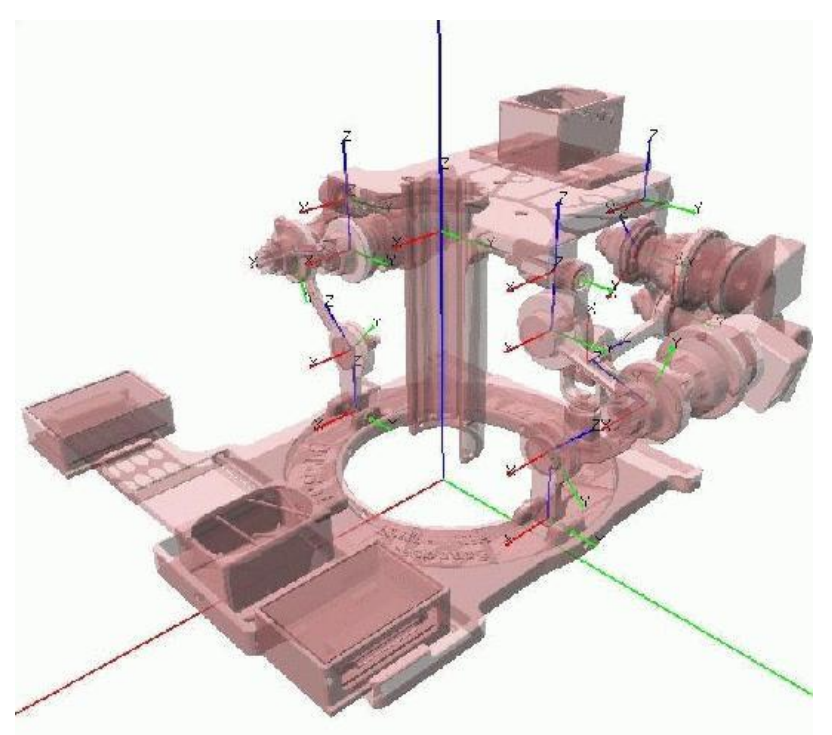

Fig. 2. CAD drawing of the system prototype

From a task point of view, the system has possibly to transmit important forces, for instance if it is equipped with a fully robotic needle driver, as presented in [12]. In that case, the robotic system has to remain motionless while the needle is inserted. The torques applied to the different joints depend on the exerted force and on the robot configuration. To insert a needle in a body a maximum force of $20 \mathrm{~N}$ might be required to allow a wide range of percutaneous interventions. From the robot Jacobian, we numerically determined that the corresponding maximum torque for a joint is $2.5 \mathrm{Nm}$.

To take into account all the constraints, including lightness and compactness, we decided to choose ultrasonic actuators. Additionally to their small size and their pretty high torque/weight ratio, the ultrasonic motors are characterized by magnetic compatibility, very low response time and also by a high holding torque that meets our safety constraint. We chose commercial Shinsei USR-30 rotary type ultrasonic motors with their power amplifiers. These motors have a limited lifetime (2000 hours for the USR-30) but can be produced at relatively low cost.

2) Position servoing: Ultrasonic motors are connected to a power amplifier that allows to control their angular velocity with an analog input. The velocity response with respect to this control signal is non-linear. The main nonlinearity is due to a dead-zone, i.e. the velocity of the motor cannot go under a minimum value ( $\pi \mathrm{rad} / \mathrm{s}$ with the USR-30). This means that in order to achieve very slow motions, the motors should work in a stepping mode.

The digital joint position control loops (one for each motor) use the angular measurements coming from incremental encoders coupled to the motors shafts and issues control signals to the amplifiers. From our experiments, it appears that a high proportional gain is sufficient to tune the position feedback loop. Indeed, since the mechanical dynamics of the motor is exceptionally fast and with no oscillatory modes, it is possible to achieve a very good rise time with no overshoot and almost no error with a simple gain ( $K=100$ in practice) even with the dead-zone. The corresponding step response diagrams are plotted on figure 3 .

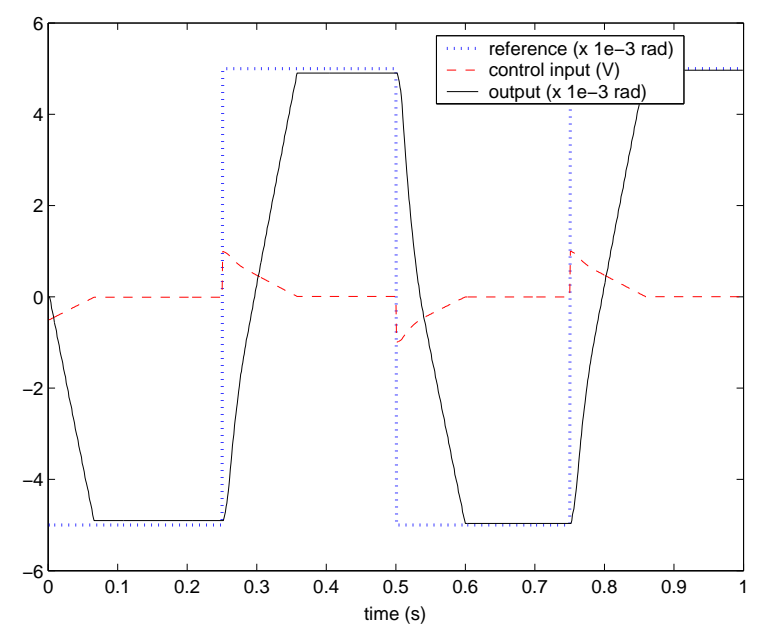

Fig. 3. Ultrasonic motor servoing with a $K=100$ proportional gain

3) Gears: To reach the required torques, the motors are associated to Harmonic Drive gears characterized by a $1 / 50$ gear ratio. The $0.05 \mathrm{~N}$ nominal torque thus allow a $2.5 \mathrm{Nm}$ torque on the output shaft. With this gear ratio the output shaft speed ranges from 0.063 to $0.586 \mathrm{rad} / \mathrm{s}$, which is quite sufficient for a medical system that has not to move fast.

Harmonic drive technology has be chosen due to their compactness and backlash-free properties. We designed a specific housing compatible with the USR-30 motor.

The association motor+gear-box fits in a $50 \mathrm{~mm} \times 30 \mathrm{~mm} \times$ $40 \mathrm{~mm}$ box which is very compact with respect to other actuation technologies like DC motors. Furthermore, with ultrasonic motors there is no need for a break or another mean that prevents any motion in case of power failure.

4) Calibration: The actuated joints are equipped with 500 increments per revolution optical encoders that need to be initialized to the correct values when the system is switched on. Several procedures can be used. The simplest solution is to use a calibrated support. The robot is placed on this geometrically precise support and the joint values are determined by inverse kinematics. An additional calibration tool such as the Polaris tracking system [17] used in the experiments is an expensive solution that has just been used for purpose of evaluation. The joint positions are stored when the system is switched off so the system needs not to be calibrated again if not necessary. 


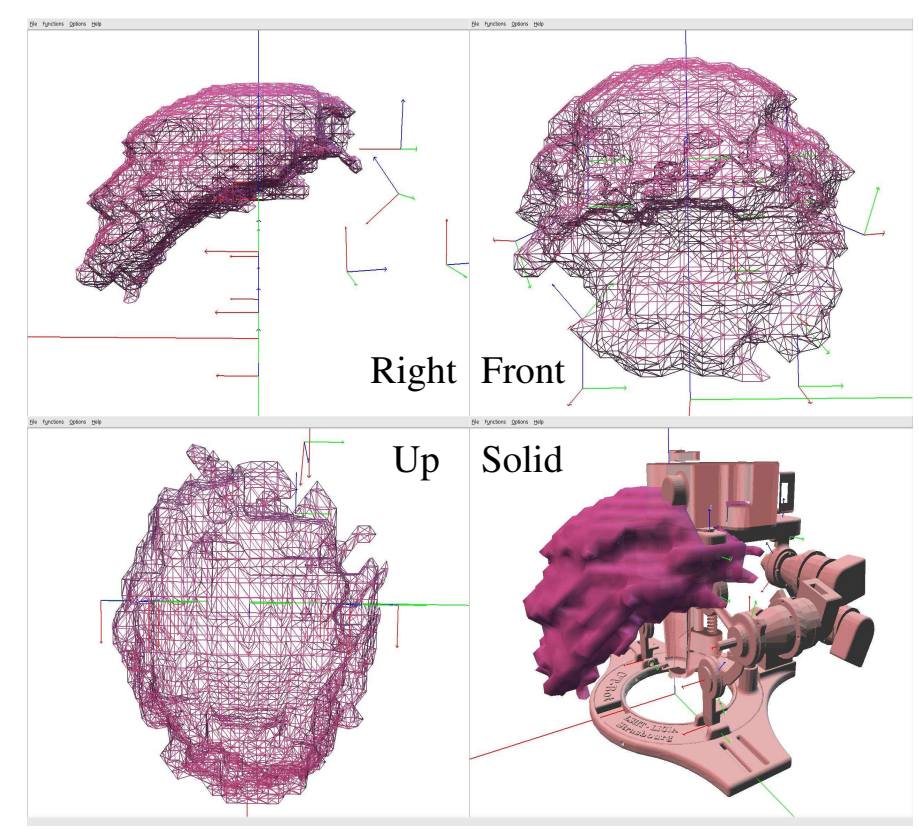

Fig. 4. Reachable position workspace of the robot

\section{Workspace and real-time collision avoidance}

Because of the robot compactness self collisions between different parts of the robot may append and it is of key importance to prevent them. This could be done during a path planning step. Nevertheless, we considered that the structure could be used in either autonomous or teleoperated modes. So, to avoid self collisions while the user is concentrated on a teleoperation task, we estimated that collisions had to be continuously checked.

The CAD model together with the OPCODE collision engine [18] were used to generate the robot configuration space for a given resolution. The discrete configuration space is stored as a 5-dimension grid where each element is boolean (inside/outside). It is then used in a real-time collision avoidance algorithm which operates at the actuator control level. On the one hand the proximity of a collision is detected and the information conveyed to the user through the user interface. On the other hand, the device is always maintained in a valid configuration and impossible motions are prevented at the joint control level.

Additionally to the collision avoidance functionality, this method allowed us to build the reachable workspace of the system and check that it is constituted by connected compact sets. The reachable position workspace is given on Fig. 4.

\section{Tasks and path planning}

The presented structure is dedicated to a positioning task (both in translation and orientation) of the tools mounted on its platform. The position is generally defined by an entry point on the patient's skin, and a target point inside the patient's body.

In the initial (calibrated) configuration the pose of the robot base is obtained by the registration of a fiducial object in the CT-scan image [16]. Fig. 5 shows the actual fiducial object which is a cube. To plan the task we developed a graphical

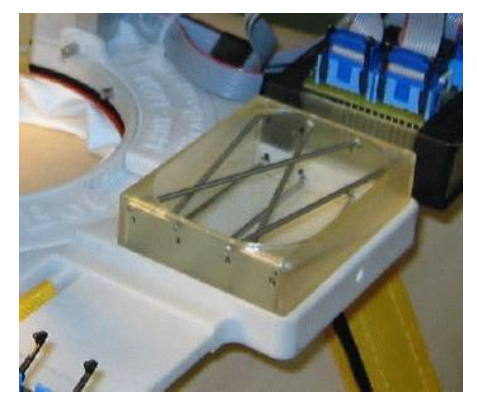

Fig. 5. The 3D fiducial cube used for experiments

user interface that makes the previous registration and then allows to define two points on the CT-scan Dicom image. From these points we compute the desired robot configuration from its inverse kinematics to achieve the corresponding positioning task. The two points define the position of an axis attached to the robot platform in the CT-scan image. It corresponds to a 5 DOF positioning and orienting task.

After this task planning, a path planning procedure is used to reach the desired configuration. This path planning is achieved by a discrete sampling method based on visibility [19].

\section{Prototype EVALUATION}

The aim of the following experiments is to evaluate the performance of the robotic prototype. To that purpose we present two different experiments. In the first one, the system is placed on a table and follows a reference trajectory. The results of this experiment give informations on the mechanical accuracy of the structure itself. In the second experiment the robot is placed in the CT-scan ring. It is registered and its ability to point a radio-opaque target is evaluated.

\section{A. First experiment}

A reference path constituted by five lines is sent to the robot controller. To evaluate both the quality of the mechanism and its joints position servo-loops, the real path of the platform is tracked with a Polaris system, which is an optical stereo tracking system [17] that allows to track simultaneously several markers with a $0.35 \mathrm{~mm}$ accuracy. A first Polaris target is affixed on the robot platform and another is placed on the robot base, as illustrated on figure 6 . The reconstruction of the relative motion of the target placed on the platform is used to compare the measured path with respect to the reference one. The results of this path tracking experiment are presented on figure 7. An error smaller than 

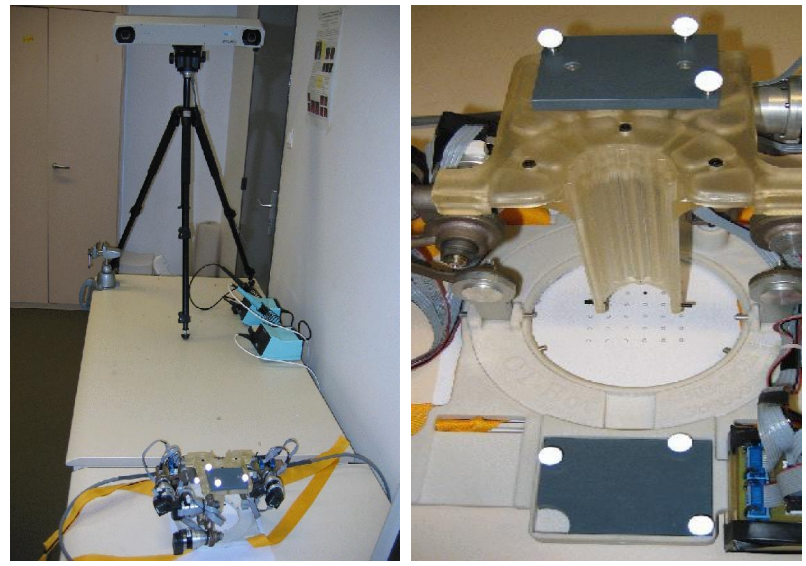

Fig. 6. Tracking of the robot motions with a Polaris : experimental setup

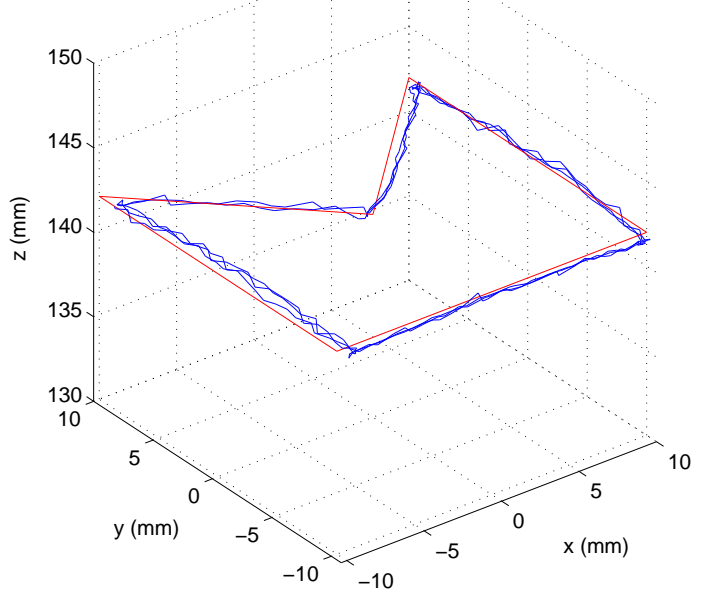

Fig. 7. Tracking of the robot motions with a Polaris : results

$2 \mathrm{~mm}$ is measured at the 5 specified points. It is compatible with our specifications since the smallest reachable structures should have $5 \mathrm{~mm}$ in diameter.

\section{B. Second experiment}

This experiment was conducted to evaluate the system for a task defined in the CT-scan image.

1) Experimental setup: The whole system (see Fig. 8) is composed of 2 PCs, the actuator power units, the robot, an abdominal phantom and a DICOM compatible CT-scan. One of the PCs is running a DICOM client program through Ethernet [20] and a graphical user interface that displays the last acquired CT image. It is connected to another PC running a real-time Linux (RTAI) robot controller thanks to a fast serial interface at $9 \mathrm{MBits} / \mathrm{s}$. The latter machine has $\mathrm{I} / \mathrm{O}$ boards that allow to control the amplifiers of the ultrasonic

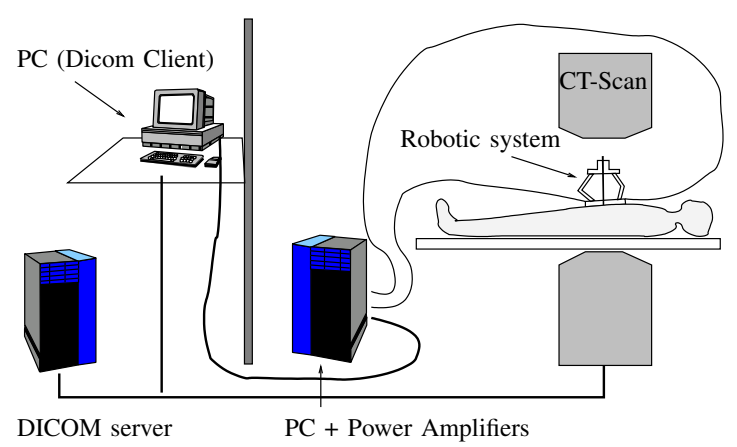

Fig. 8. Overall setup

motors.

2) Pointing task: The system is used to point a target with a laser source. First, the system is automatically registered thanks to the projection of the fiducial object in the Dicom image (cf. Fig. 9 and [16]). An average root mean square registration error of 0.09 pixels, i.e. $0.045 \mathrm{~mm}$ for each spot centroid location is obtained.

The user interface is then used to choose a first point on the CT-scan image which corresponds to a possible entry point on the patient and a second point, which corresponds to the target point in the patient's body (cf. Fig. 9). This target point is materialized by a small radio-opaque target which size is 5 by $3 \mathrm{~mm}$ in order to be visible in at least one CT-scan slice (cf. Fig. 9).

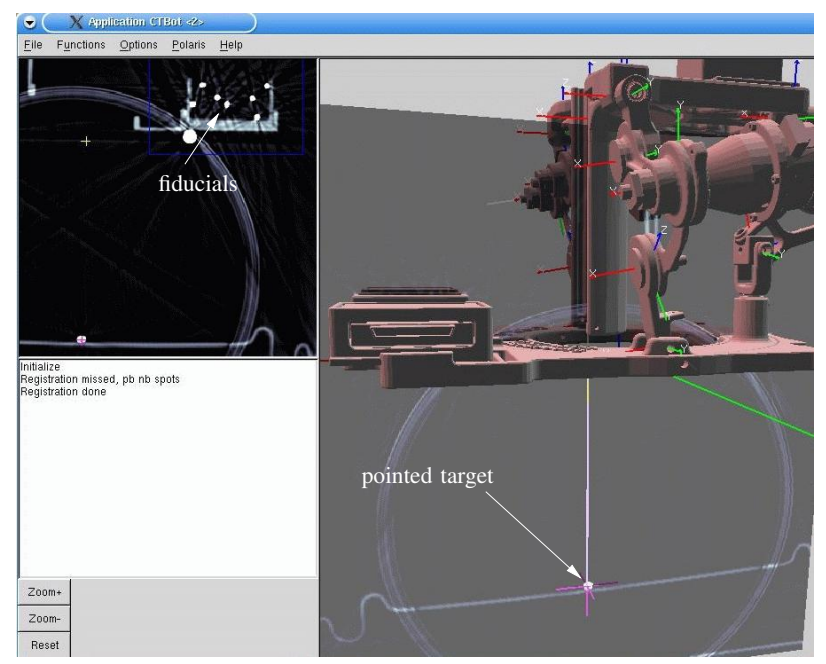

Fig. 9. Task path planing

With a laser source mounted on the robot platform the result is visually checked. This allows to evaluate the mechanical accuracy. If the target had been pointed with a needle, the influence of the phantom and the needle bending could not have been distinguished from the mechanism and registration accuracy. The projection of the laser spot on the target has an average accuracy of less than $3 \mathrm{~mm}$ (cf. Fig. 
10), for a platform-target distance ranging from 200 to 250 $\mathrm{mm}$.

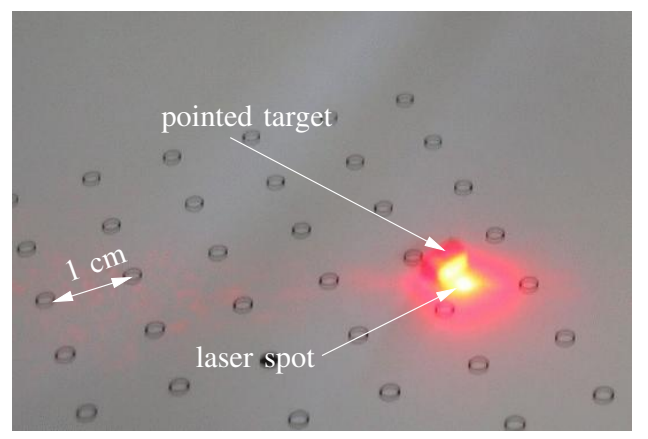

Fig. 10. Laser pointing $(\mathrm{depth}=250 \mathrm{~mm})$

\section{Conclusion}

In this paper we detail the different steps that have been necessary to design and build a robot adapted to the conditions of the operating room. From the concept of a parallel structure placed on the patient's abdomen, we point out the various practical issues that have to be taken into account specifically for such a system : compactness, intrinsic safety of the actuators, high forces, high accuracy. In our opinion, these points have a certain generality and can offer solutions for a system sharing the same constraints.

Since the resulting prototype is far different from standard manipulation solutions we evaluated its ability to fulfill the precision requirements. Absolute position tracking was evaluated from an external measurement system. Finally, the ability of the system to point a target under CT-scan guidance was validated and a precision of $3 \mathrm{~mm}$ was observed for a typical intervention depth.

Perspectives include the evaluation of this system in the context of a needle insertion task. The influence of the needle bending that has not been treated in this paper will then have to be considered. In the perspective of automated or teleoperated needle insertions, the design and manufacturing of a dedicated needle driver will be another future direction of investigation.

\section{ACKNOWLEDGMENT}

This work has been supported by the Alsace Region Council and the French CNRS ROBEA (Robotics and Articifial Entities) program.

\section{REFERENCES}

[1] A. Gangi and J.-L. Dietemann, Tomodensimétrie Interventionnelle. Editions Vigot, PARIS, 1994.

[2] S. Silverman, K. Tuncali, D. Adams, R. Nawfel, K. Zou, and P. Judy, "CT fluoroscopy-guided abdominal interventions: Techniques, results, and radiation exposure," Radiology, no. 212, pp. 673-681, 1999.

[3] Y. S. Kwoh, J. Hou, E. Jonckheere, and S. Hayati, "A robot with improved absolute positioning accuracy for CT guided stereotactic brain surgery," IEEE Transactions on Biomedical Engineering, vol. 35, pp. 153-160, February 1988.
[4] D. Stoianovici, L. L. Whitcomb, J. H. Anderson, R. H. Taylor, and L. R. Kavoussi, "A modular surgical robotic system for image guided percutaneous procedures," in Proceedings of the 1998 International Conference on Medical Image Computing and Computer-Assisted Intervention, (Cambridge, MA), MICCAI'98, October 1998.

[5] N. Navab, B. Bascle, M. H. Loser, B. Geiger, and R. H. Taylor, "Visual servoing for automatic and uncalibrated needle placement for percutaneous procedures," in Proceedings of the IEEE International Conference on Computer Vision and Pattern Recognition, vol. 2, pp. 2327-2334, CVPR 2000, June 2000.

[6] K. Cleary, M. Freedman, M. Clifford, D. Lindisch, S. Onda, and L. Jiang, "Image-guided robotic delivery system for precise placement of therapeutic agents," Journal of Controlled Release, vol. 74, pp. 363368, 2001.

[7] K. Masamune, G. Fichtinger, A. Patriciu, R. C. Susil, R. H. Taylor, L. R. Kavoussi, J. H. Anderson, I. Sakuma, T. Dohi, and D. Stoianovici, "System for robotically assisted percutaneous procedures with computer tomography guidance," Computer Aided Surgery, vol. 6, pp. 370 $-383,2001$.

[8] S. Lavallee, J. Troccaz, L. Gaborit, P. Cinquin, A. Benabid, and D. Hoffmann, Computer Integrated Surgery, ch. Image-Guided Operating Robot: A Clinical Application in Stereotactic Surgery, pp. 343-352. MIT Press, 1995.

[9] J. Hong, T. Dohi, M. Hashizume, K. Konishi, and N. Hata, "An ultrasound-driven needle-insertion robot for percutaneous cholecystostomy," Physics in Medicine and Biology, pp. 441-455, 2004. IOP Publishing Ltd.

[10] P. J. Berkelman, P. Cinquin, J. Troccaz, J.-M. Ayoubi, C. Ltoublon, and F. Bouchard, "A compact, compliant laparoscopic endoscope manipulator," in Proceedings of the 2002 IEEE International Conference on Robotics and Automation, (Washington DC, USA), pp. 1870-1875, ICRA'02, May 2002

[11] A. Vilchis, J. Troccaz, P. Cinquin, K. Masuda, and F. Pelissier, "A new robot architecture for tele-echography," IEEE Transactions on Robotics and Automation, vol. 19, pp. 922-926, October 2003.

[12] B. Maurin, O. Piccin, B. Bayle, J. Gangloff, M. de Mathelin, L. Soler, and A. Gangi, "A new robotic system for CT-guided percutaneous procedures with haptic feedback," in Proceedings of the 2004 Computer Assisted Radiology and Surgery Congress, (Chicago, USA), CARS'04, June 2004.

[13] B. Maurin, O. Piccin, B. Bayle, J. Gangloff, and M. de Mathelin, "A parallel 5 dof positioner for semi-spherical workspaces," in Proceedings of the 2004 ASME Design Engineering Technical Conferences (in press), (Salt Lake City, USA), DETC'04, October 2004.

[14] R. C. Susil, J. H. Anderson, and R. H. Taylor, "A single image registration method for ct guided interventions," in Proceedings of the International Conference on Medical Image Computing and ComputerAssisted Intervention (MICCAI 99), (Cambridge, United Kingdom), pp. 798-808, 1999.

[15] S. Lee, G. Fichtinger, and G. S. Chirikjian, "Numerical algorithms for spatial registration of line fiducials from cross-sectional images," American Association of Physicists in Medicine, vol. 29, pp. 18811891, August 2002.

[16] B. Maurin, C. Doignon, J. Gangloff, B. Bayle, M. de Mathelin, O. Piccin, and A. Gangi, "Ct-bot: A stereotactic-guided robotic assistant for percutaneous procedures of the abdomen," in Proceedings of the 2005 SPIE Conference on Medical Imaging, (San Diego, USA), MI'05, fvrier 2005.

[17] NDI Polaris System, http://www.ndigital.com/polaris.php.

[18] P. Terdiman, "Opcode - optimized collision detection." http://www.codercorner.com/Opcode.htm.

[19] T. Simeon, J.-P. Laumond, and C. Nissoux, "Visibility based probabilistic roadmaps for motion planning," Advanced Robotics, vol. 14, no. $6,2000$.

[20] Dicom@Offis, “Dcmtk 3.5.3 - dicom toolkit." http://dicom.offis.de/dcmtk.php.en. 